enficient air mas stop the reppirition. I have ceen two cases, both occurring in elderly females, in whom suffocation nearly occurred from the tongue falling back into the tion nearly occurred fres. The breathing was suspended in both cases, and the simple pulling forward of the tongue was followed by tong inspiration. This was pointed out to me by Mr. Fdward R. Bickersteth.

In young children, the administration of chloroform is an anxious duty, and requires the greatest care. Very little should be put on the fold of lint, and it should be rery carefully held two or three inches from the mouth and nose. No attempt should be made to get them very quickly under the influence. They seem to be peculiarly susceptible of its poisonous influence, and these are cases in which $I$ believe it really does kill by absorption, and by thus producing syncope ; for often after the operation is over, and the chloroform removed, young children fall into a state of very deep insensibility, and we are alarmed to find the pulse falling, and the colour deserting the face. In these cases, I have found that raising the feet to a higher level than the chest, together with the application of warmth to the body, have the best effect; and the access of cool air to the face and lungs is essential.

That vomiting should be a frequent accompaniment of insensibility from chloroform need not surprise us, when we consider the close sympathy established by the pneumogastric nerve between the lungs and the stomach, which seems, in a great measure, to explain the effects of ipecacuanha, squills, etc.; also the effect of various states of the stomach in inducing fits of asthma. Vomiting is easily prevented by prohibiting the taking of any food for three or four hours previous to the operation.

I shall be very glad if my drawing attention to this subject shall tend to cause greater care to be taken in the administration of chloroform; as otherwise I feel convinced, that deaths from it will be of no unfrequent occurrence, and will strengthen the prejudices that some still retain against its use.

1, Caxton Terrace, St. James's Road, Liverpool, March 1854.

\section{THE NEGLECT OF THE USE OF BLEEDING IN THE TREATMENT OF SOME OF THE MILDER AILMENTS.}

\section{By J. A. HINGESTON, Esq.}

ErER since the publication of Dr. Marshall Hall's excellent remarks on the danger of excessive blood-letting, many years ago, there seems to have prevailed among English practitioners a reverential horror of the loss of blood. Diseases are treated as successfully in the present day without the loss of blood, as they used to be formerly with the almost unrestrained use of the lancet. This decided change of practice is brought forward as a triumphant proof of the improvement in the treatment of disease, whereby a cure is effected without having recourse to those remedies which drain off the force of the vital fluid. It is affirmed, that the general health suffers considerably less under this milder mode of management than it did under the more heroic practice of our predecessors.

Granting that there is a great deal of sound truth and reason in the foregoing remarks, $I$, for one, cannot give my entire consent to the conclusion legitimately deducible from them. I was born and bred in the practice of medicine. I can look back upon an interval of thirty-five years at least, during which period this decided change of practice has taken place. I can remember the time when bleeding was, as it is styled, "the order of the day"; and I own, that on calling to mind many cases in which this practice was adopted, it appears to me, at this distance of time and at my present mature age, to have been sometimes both unnecessary and, perhaps to a certain degree, injurious. It was, however, the practice then in rogue, just as the non-bleeding practice is the one most in vogue now. It received the common-consent of the beat practitionem; and, in this sense alone, it was certainly entitled to our reapect.

What is most approred of for the time being, is upon the whole the best. The character of disease has altered. Nervous diseases prevail now in the manner as the phlogistic maladies prevailed up to the year 1832. The latter demanded frequent loss of blood, whereas the former are rendered worse by bleeding, but are cured by tonics and a generous diet. It is probable that many nervous diseases were overlooked during the phlogistic period, just the same as that phlogistic diseases are overlooked by the practitioners of the present day. There is nothing to congratulate ourselves upon; for, if we act prudently in withholding the lancet now, our predecessors acted quite as prudently in making use of it formerly.

Taking this view of disease, we must allow that a great deal may be said in favour of both kinds of practice. Truth generally lies half way between the disputants. What I am contending for is, that we are disposed to look too much on one side of the question only, and are apt to lay aside the use of the lancet in the ordinary course of practice, to the prejudice, as it appears to me, of the beneficial and successful treatment of many of the milder ailments.

I have heard practitioners boast of never carrying their lancets with them, and of being able to treat almost every illness that comes before them without ever being forced to have recourse to it. In short, they declare that their lancets are next to useless. Without questioning their ability in the management of disease by means of remedies much less obnoxious than that of blood-letting, we must at least be permitted to declare that such a boast as this is not merely an idle one, but it also shows that they are wilfully resolved to exclude as far as they can one of the grand agents in the practice of medicine.

To bleed properly, requires a good deal of time. It delays. the routineer in the progrees of his round. It cannot be dismissed in a few minutes. It imposes the delay of an hour or more. Some time must be spent in watching the flow of the blood, and its immediate effects on the constitution. If the patient should faint, the delay is still longer. I am inclined to think that the trouble of bleeding is one of the chief reasons for omitting it, whenever this can be done with safety.

The use of blood-letting is very great on many more occasions than those of acute inflammation, in which it is absolutely unavoidable. Many diseases, or even ailments, are rendered much more manageable by premising the general treatment with a single venesection. It is not the quantity lost-it is the effect produced. A large quantity is not requisite. A small quantity will frequently relieve in the most unequivocal manner. A teacupful, for instance, or only half as much, taken away in a full stream, will ameliorate all the symptoms, afford a refreshing sleep, cause the skin to perspire, and the bowels to relax, increase the appetite, and give a feeling of relief and ease which no other remedy or any mode of diet is able to supply. This happy result is more particularly the case, when the pulse has been found contracted, suppressed, or jerking. It will occasionally decide the cure, and scarcely any medicine is needed afterwards. Moreover, if we are in doubt as to the propriety of blood-letting, a small quantity taken away in a deep cup, and allowed to stand apart for twelve hours or so, will afford us an opportunity of inspecting the quality of the blood, and of informing ourselves of the exact state of the constitutional diathesis.

In all cases of local fulness of blood, bleeding ought not to be omitted, either by leeches or the lancet. In cases of debility even, it is excellent practice to take away blood, and to give steel at the same time. The shock sustained from a violent blow or concussion, hereditary plethora, venous torpidity, visceral congestions, etc., are each of them relieved, if not cured, by a judicious loss of blood. Sedentary habits, a good deal of in-door occupation, and the close confinement of certain trades, lead to many of those passive derangements of the health, which field to venesection, 
Glin hratives, and the bitters, conjoined with fresh air, much more speedily than they would do if the bleeding clone were omitted.

It is true, that ailments of this kind may be accounted for upon the principle of shoer debility-asthenia, as it is termed, and may be cured by tonics, at the head of which are the combinations of iron. But, most likely, acute inflammation, which incontestably demands the loss of blood without delay, is the last stage of debility, either local or general; and the over-distended vessels recover their power of contracting themselves to their natural calibre, simply by being relieved of their superabundant load of blood. Possibly, this may be the real pathology of acute inflammation; and the treatment which cures inflammation may, upon the same principle, be equally applicable to the debility which is the prime cause of inflammation itself. Be this as it may, it is certain that excessive fatigue, or over-exertion of the head and chest, tends to congestion of the brain, lungs, liver, abdomen, and lower extremities, which is best treated by a moderate venesection, appropriate to the age, sex, stature, strength, and habits of the patient. As a general rule, it may be affirmed, that those patient. As the fresh air and enjoy robust health, require bleeding much less than those who reside in the close atmosphere of large towns.

To bleed well is not always an accomplishment of the professed surgeon. Perhaps, there are not many who may be styled good bleeders. It is a very nice operation, and requires a good deal of practice, as well as study and attention. The mode of performing this commonplace operation might deserve a little treatise by itself. To excel in all its details imparts an air of gracefulness to the skill of an ordinary practitioner.

I am not speaking here of that bold depletion practised on many important and peremptory occasions; such as apoplexy, the reduction of a dislocated limb together with the use of tartar emetic, puerperal convulsions, peritonitis, etc., when it is necessary to take away blood to faintness, nausea, vomiting, or syncope,-till the sight grows dim, the ears whiz, the face is blanched, and death is apparently not far off. I am not speaking of extreme cases, such as those in which we are forced to make our choice between two terrible evils, - certain death from the disease or accident, or contingent death from the use of a severe remedy. What I wish to bring before the notice of the medical profession, is the use of moderate bleeding in the treatment of some of the milder ailments. I will endeavour to point out a few of them.

Simple fulness (plethora) with precordial distress, a large pulse, deranged liver, and a loaded white tongue, is best treated by bleeding, calomel, and salines. Most likely, in the course of twenty-four hours, the feeling of health is ro-established, and no further medical attention is required. Omit the bleeding, and indisposition lingers on for a week or ten days, or perhaps what is called gastric fever supervenes. Again, when the hæmorrhoidal vessels become overcharged, and are seen projecting from the anus, a single bleeding will restore the balance of the circulation, and recovery is at once assured. There is a form of indigestion which yields neither to tonics nor alteratives, and is not eren benefited by change of air. The loss of appetite, or the distress following a full meal, is as rexatious as it is obstinate. The tongue is clean, except at the root; and the breath is hot and offensive. Bleeding, and a blister to the pit of the stomach, put all to rights. Constipation is another form of dyspepsia, which, when it is not habitual, should never be treated without venesection. For the most part, when constipation happens unexpectedly, and continues with obstinacy, without any reason for suspecting mechanical obstruction, hernia, or intussusception, there is lurking some degree of inflammation in some of the textures. The blood is generally cupped and buffed. Headaches, spinal pains, pains in the loins, diminished excretion of urine, and uncasy respiration, accompanied with deep aighing, although auscultation afiords no evidence of disane, aro all of them ailments in which blood-letting is called for in limine. Brysipelas, so frequently treated with tonics and stimulants, is a milder illness when reliered by blood-letting at the first. Rheumatism, both acute and chronic, though never cured by decided and repested loss of blood, is a much more manageable disease when the thet any one watch the course of chronic rheumatism, left to take its one watch the course of the natural result of it is in the restoration of health: it is epistaxis, or a phlegmonous abscess, or a sharp attack of inflammatory fever. Nature, in the end, points out the plan which it would have been wiser to have acted upon in the beginning. In the very werinning of fevers, a single bleeding, together with an emetic, was the wise practice of former days, although it is scarcely ever thought of now. In all paralytic affections, passive or acute, bleeding, either locally or generally, phould never be omitted. In cerebral attacks, whether should never be omitted. In cerebral attacks, whether those cases do the best in which the loss of blood has been full, early, and energetic. I declare I have never regretted having taken away blood at the onset in these cases; but I have had occasion to be sorry for baving omitted it when have had occasion to be sorryled by my superiors, or when I have not seen my way clearly at first starting. In sporadic epilepsy, bleeding is injurious. It is supposed that some of the convulsive forms of cerebral disease arise from a contaminated condition of the blood, and that, in cases where the blood is poisoned, as it were, bleeding can only add to the debility already present. In my humble opinion, this notion is groundless. To me it appears, on the contrary, the very reason why loss of blood is demanded. It is best to get rid of the contaminated fluid as quickly as possible; for it gives the vis medicatrix the opportunity of creating anew a fresh supply of better blood, if the patient be forthwith placed in a position favourable to the healthy process. And thus again, paradoxical as it may sound, anæmia, or the want of blood, is well treated by bleeding and tonics conjoined-repeated small bleedings, and a very generous table at the same time. The sanguification and assimilation are singularly stimulated to resume a healthier mode of existence; the blood, which was at first brown and watery, becomes by degrees rich and florid; and the tongue is no longer flabby and sodden, nor are the lips and cheeks so continually wanting in their tints of carnation. The eye sparkles once more, and the hair curls. The age of puberty in both sexes, but particularly in boys, is marked by a sudden turgescence of the animal system. The head suffers a good deal, the eye is bloodshot occasionally, and there is a tendency to impulsive acts and eccentric emotions. Bleeding is the best and the only remedy in these cases. It will ward off many an evil, both moral and physical, which otherwise might damage the character and health very seriously. Death from a short fever and effusion on the seriously. Death is one of the catastrophes at this critical epoch pointing out, as clearly as possible, the direction of the treatment we ought to pursue. A sudden chill, or a sudden shock to the nerves in consequence of a fall or injury, calls for the loss of blood as soon as the constitution has recovered itself, and reaction sets in.

I have pointed out a very few of the cases in which bleeding is serviceable, and in which I think I perceive that, from the prevailing notions of the day, almost exclusively opposed to depletion, blood-letting is not so frequently practised as it ought to be.

I have no wish to work a single idea threadbare. Judgment and discretion are as requisite here as they are in any of the other affairs of life; and I submit these few brief remarks to the consideration of those who are as much above me in the esteem of the world as they are in their professional rank, talents, and experience.

Brighton, March 1854. 